\title{
Pierce the ear and stab the spleen
}

Thomas Matteazzi, Italo Balzani, Michele Alberghini, Federica Beretta, Claudia Bon, Davide Dotto, Martina Frigo, Olga Ilinciuc, Flavia Lanzuisi, Sara Angela Malerba, Mauro Stragliotto, Dorian Vataj, Elena Campello

Internal Medicine, Azienda Ospedale Università, Sant'Antonio Hospital, Padua, Italy

\begin{abstract}
Splenic abscess is a rare but extremely dangerous condition generally spreading from a local, or systemic, focus of infection. We present the case of a young immunocompetent female admitted with sepsis and multiple splenic abscesses. The patient had a recent left ear piercing on the tragus complicated by an ear infection. The presence of a solitary parotid abscess, the absence of other infectious foci on computed tomography scan, the negativity of blood cultures and the absence of endocarditis vegetations led us to think that the most likely culprit was a hematogenous dissemination from the left tragus. The patient was successfully treated with intravenous antibiotics. There had been no need of splenectomy or any other procedure. This rather unique case underscores that splenic abscess should be suspected when a long-lasting fever and pain in the left hypochondrium are present, even when an apparently innocuous invasive procedure, such as a body piercing, is performed.
\end{abstract}

\section{Introduction}

Splenic abscess is a rare condition with an estimated frequency of $0.05-0.7 \%$, and documented evidence of 600 cases in the literature. There are many predisposing factors such as immunodeficiency, bacteremia secondary to trauma, embolization or hemoglobinopathies. Splenic abscess generally spreads from a local, or systemic, focus of infection. ${ }^{1}$

We present the case of a young immunocompetent female admitted with sepsis and multiple splenic abscesses. The patient had a recent left ear piercing complicated by an ear infection. She was successfully treated with intravenous antibiotics. There had been no need of splenectomy or any other procedures.

Correspondence: Elena Campello, Internal Medicine, Azienda Ospedale Università, Sant'Antonio Hospital, via Giustiniani 2, Padova, Italy.

Tel.: +39.049.8216444 - Fax: +39.049.8216445.

E-mail: elena.campello@aopd.veneto.it

Key words: Antibiotics; cervical lymphadenopathy; infection sepsis; spleen abscess.

Conflict of interests: the authors declare no conflict of interests.

Received for publication: 29 January 2020.

Accepted for publication: 4 February 2020.

This work is licensed under a Creative Commons Attribution NonCommercial 4.0 License (CC BY-NC 4.0).

${ }^{\circ}$ Copyright: the Author(s), 2020

Licensee PAGEPress, Italy

Italian Journal of Medicine 2020; 14:95-99

doi:10.4081/itjm.2020.1263

\section{Case Report}

A 17-year-old female presented to our emergency department (ED) with fever, left cervical lymphadenopathy and left tragus edema. Six months earlier she had a piercing on the same ear. She was discharged from the ED following an Ear, Nose and Throat (ENT) specialist examination, with a prescription of antibiotic therapy (azithromycin) for a left ear abscess. The patient returned to the ED a few days later with high fever and abdominal pain, and thus she was admitted to our Medical Unit. The patient's past medical history included an inflammatory bowel disease - ulcerative proctitis - diagnosed four months earlier. She was on oral mesalazine.

Upon physical examination, her blood pressure was $120 / 70 \mathrm{mmHg}$, pulse rate was 104 beats per minute and temperature was $37.5^{\circ} \mathrm{C}$. There was a hard and painful palpable lump in the left parotid gland and the left posterior auricular region. Chest auscultation revealed no evidence of heart murmurs or pathological pulmonary sounds. There was mild abdominal tenderness with evident pain when pressing the left quadrants, positive costovertebral angle tenderness (Giordano's test) on the left side, negative Blumberg's sign and no splenomegaly, unobstructed bowel without diarrhea. Chest X-rays showed no pathologic signs. During the first days of hospitalization body temperature was characterized by feverish peaks, up to $39^{\circ} \mathrm{C}$, during which the left flank pain worsened.

Serum markers of inflammation and infection were above the normal range: white blood cells (WBCs) $14,300 \times 10^{9} / \mathrm{L}$ with neutrophilia (82\%), C-reactive protein (CRP) $98 \mathrm{mg} / \mathrm{L}(\mathrm{NR}$, normal range $<3.0 \mathrm{mg} / \mathrm{L}$ ), erythrocyte sedimentation rate (ESR) $64 \mathrm{~mm} / \mathrm{h}$ (NR $<20 \mathrm{~mm} / \mathrm{h}$ ), procalcitonin $1.55 \mathrm{mcg} / \mathrm{L}(\mathrm{NR}<0.5$ 
$\mathrm{mcg} / \mathrm{L})$. Renal and hepatic function markers were within the normal range. Blood and urine cultures were collected before initiating antibiotics - no bacteria were detected. Serologic tests for Epstein-Barr virus, Cytomegalovirus and Bartonella spp were all negative. Similarly, quantiferon and anti-nuclear antibodies (ANA) tests were also negative. A broad spectrum combined antibiotic therapy with piperacillin-tazobactam and ciprofloxacin was initiated.

A neck ultrasound revealed a $25 \times 14 \mathrm{~mm}$ hypoechoic formation, with no vascular signs in the left parotid gland compatible with colliquated lymph nodes; other reactive lymph nodes were found during the ultrasound examination.

An abdominal ultrasound was performed revealing three marginal and hypoechoic formations in the spleen of not univocal interpretation (Figure 1A-C).

An abdominal CT (Figure 1D-F) was therefore performed. A mild splenic enlargement (bipolar diameter, $\varnothing 13 \mathrm{~cm}$ ) and four sub-capsular hypodense lesions ( $\varnothing 1$ $\mathrm{cm}$ each) were detected; no enlarged lymph nodes were found. A trans-thoracic cardiac ultrasound excluded the

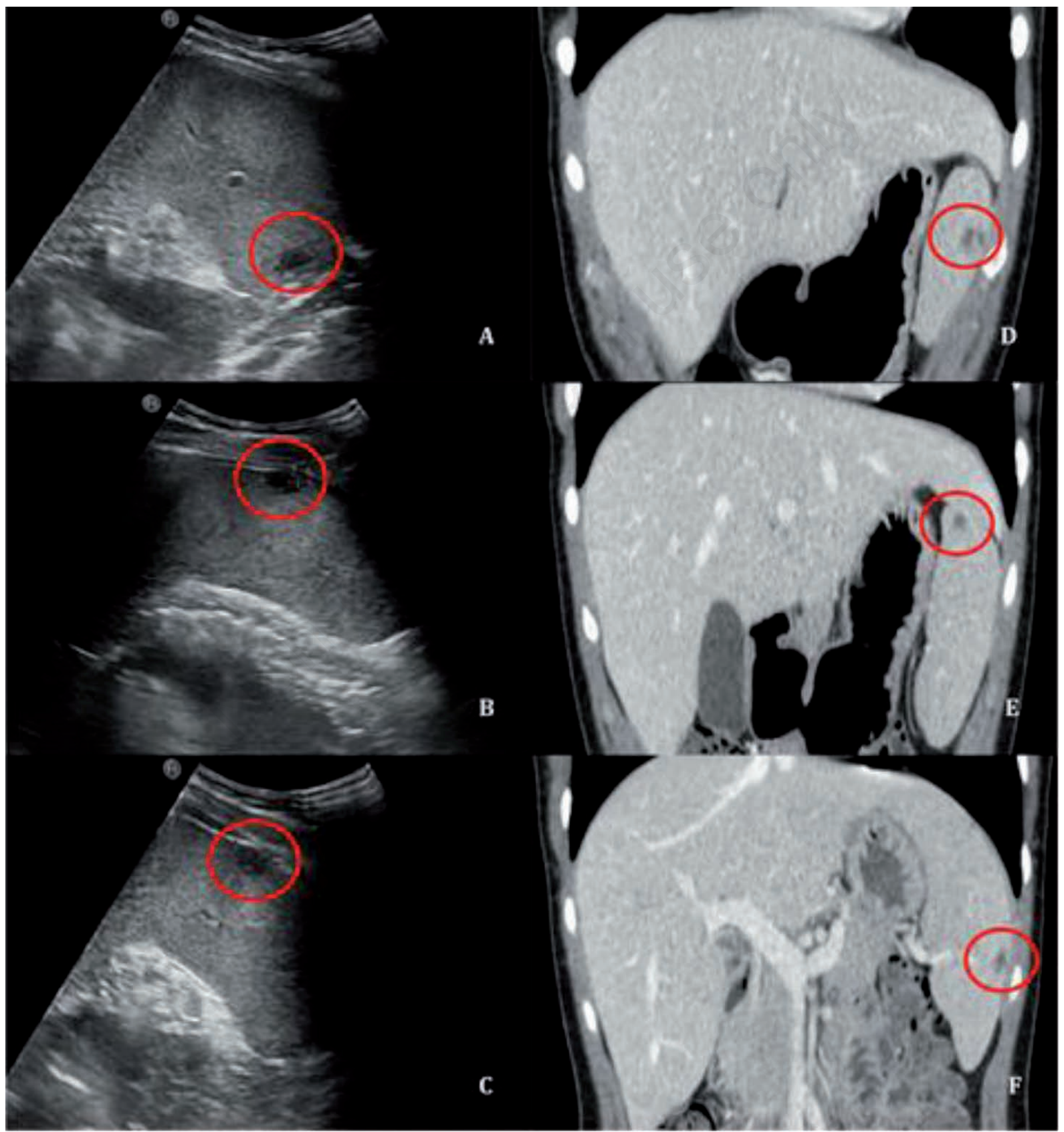

Figure 1. A-C) Ultrasound images of the spleen lesions (inside red circles) at hospital admission; D-F) computed tomography images of the spleen lesions (inside red circles). 
presence of endocarditis. We also decided to perform a cervical magnetic resonance imaging (MRI) to better characterize the suspicious colliquated parotid lymph node. The MRI (Figure 2A and B) revealed two oval formations in the left parotid gland, the biggest of which presenting a hyperintense and liquid central area. An ultrasound-guided fine needle aspiration (FNA) brought about purulent material with negative cultures. Antibiotic therapy produced an improvement of the clinical parameters and decline in serum markers of inflammation. After two weeks of therapy an abdomen ultrasound showed stability of splenic lesions. The patient was discharged with a prescription of oral antibiotics (amoxicillin/clavulanic acid). She completed a four-week therapy without complications. An abdomen CT scan was performed and confirmed the complete resolution of all splenic lesions.

\section{Discussion}

This rather unique case underscores the difficulties in diagnosing spleen abscess (Table 1). The formation of splenic abscess usually follows one of the following three mechanisms: i) hematogenous dissemination during sepsis (typical of multiple abscesses); ii) the intrinsic theory, that is, an infection as a consequence of an alteration of the splenic structure, as it is the case in hematomas, splenic infarcts and hemoglobinopathies; iii) the extrinsic theory (typical of single

Table 1. Characteristic aspects of splenic abscess.

\begin{tabular}{ll}
\hline Spleen abscesses: etiological, clinical, radiologic features and treatment regimens \\
\hline Etiological agents & - Bacterial: Gram positive cocci (Streptococci and Staphylococci spp), Enterobacteria, Anaerobes spp \\
& - Fungal: C. albicans, Aspergillus spp, Cryptococcus spp, Pneumocystis carinii Parasitic: Echinococcus granulosus \\
\hline Clinical features & - Classic triad: fever, left hypochondrium pain and palpable mass (36\% of cases) \\
& - Non-specific signs: splenomegaly, nausea, weight loss, non-specific abdominal pain (40-54\% of cases) \\
\hline Imaging & - CT with contrast agent (gold standard) \\
\hline Differential diagnoses & - Splenic neoplasms: lymphomas, benign and malignant vascular neoplasms, hamartomas \\
& - Splenic vascular lesions: splenic infarcts, peliosis hepatis, Gamna-Gandy bodies (portal hypertension) \\
\hline Treatment options & - Antibiotic therapy + CT-guided drainage (solitary lesions with homogeneous content) \\
& - Antibiotic therapy alone \\
\hline Antibiotic regimens & - Splenectomy (multiple abscesses) \\
& - Fluoroms + metronidazole for 3-4 weeks \\
\hline
\end{tabular}

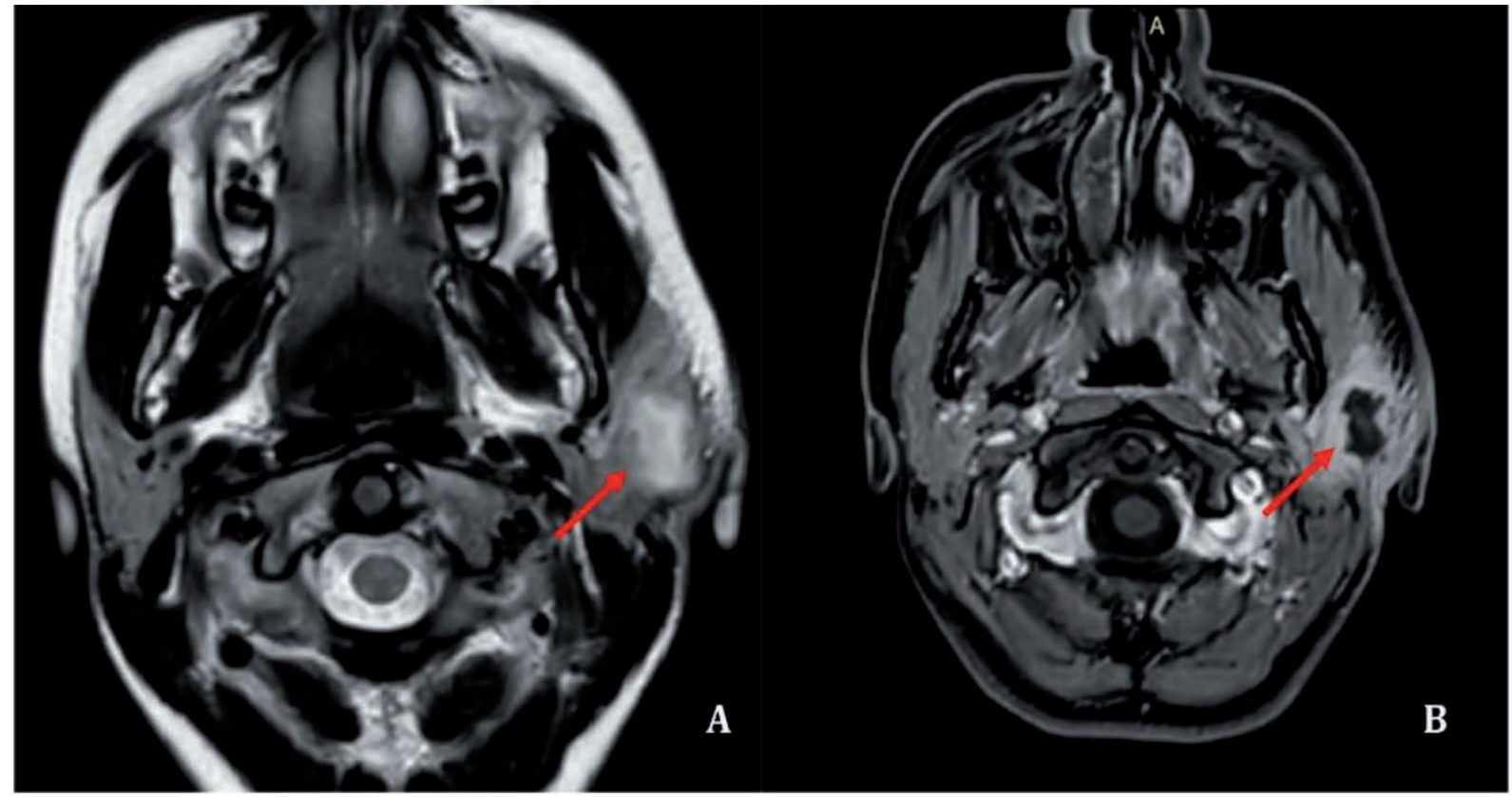

Figure 2. A and B) Magnetic resonance images of the parotid abscess (red arrows). 
abscess), that is, a consequence of a local diffusion spreading from a contiguous infectious focus. ${ }^{1}$ The solitary parotid abscess, the absence of other infectious foci on CT, the negativity of blood cultures and the absence of endocarditic vegetations led us to think that the most likely culprit, in our case, was a hematogenous dissemination. Furthermore, the diagnosis was made even more challenging because of the absence of risk factors such as immunosuppression, intravenous drug use, alcohol abuse, chronic liver diseases and bacteremia secondary to trauma. ${ }^{2-5}$

The median time of diagnosis in the literature ranges from two to four weeks. ${ }^{6}$ In our case, because of the aggressive diagnostic work-up, it took only five days.

The patient presented with two out of the three characteristic symptoms of the triad of Sarr and Zuidema: pain in the left hypochondrium and fever. However, there was no evidence of a palpable splenic mass. ${ }^{7}$ Other non-specific signs of this disease such as nausea, neutrophilic leukocytosis and diffuse mild abdominal pain were present. ${ }^{8}$

The microorganisms most frequently responsible for splenic abscess are Gram-positive cocci (Staphylococci and Streptococci), enterobacteria (especially salmonella) and anaerobes. Fungal abscesses are typically caused by Candida albicans in immunocompromised patients. ${ }^{1}$ In our case, both culture and serologic tests were inconclusive. This particular circumstance finds little corroboration in the literature, with the exception of a report by Mushtaq et al. who were unable to isolate a responsible microorganism. ${ }^{5}$ The diagnostic value of blood cultures may vary from $24 \%$ to $80 \%$ and positive cultures are linked to the presence of multiple abscesses. Furthermore, cultures of the drainage fluid appear to be much more sensitive albeit burdened by a higher complication rate due to the procedure. ${ }^{9}$ In our case the blood cultures negativity may be explained by the previous rounds of antibiotic therapy.

Another obstacle in the diagnostic process lies in the interpretation of X-rays which may be not univocal. CT scan remains the gold standard for diagnosis with a $95 \%$ sensitivity and $92 \%$ specificity. ${ }^{1}$ The abscess can be solitary, multiple or with multiple loculi. Multiple lesions are typically small $(\varnothing 5-10 \mathrm{~mm})$, centrally located, round or irregular in shape and hypodense in the center. ${ }^{10}$ Although the presence of gas is a diagnostic marker, it can be found only in a few cases. ${ }^{11}$ The lesions in our case were about $\varnothing 1 \mathrm{~cm}$ and located in the peripheral sub-capsular region.

The differential radiological diagnosis also raised a few issues: splenic abscesses may present differently depending on the infectious agent - bacterial, mycobacterial, fungal, echinococcal or pneumocystis. Differential diagnosis for splenic lesions also includes splenic neoplasms (e.g., lymphomas, benign and ma- lignant vascular neoplasms, hamartomas) and vascular lesions (e.g., splenic infarcts, peliosis hepatis and Gamna-Gandy bodies from portal hypertension). ${ }^{11}$

As for treatment, there is still no gold standard. The treatment of choice is often total splenectomy, combined with antibiotic therapy, though antibiotic therapy alone has been proven effective in some cases. ${ }^{12}$ There are no studies regarding specific antibiotic regimens. The little data available derive from studies conducted on patients admitted to surgical departments for intra-abdominal infections who were successfully treated with beta-lactams and/or fluoroquinolones ${ }^{1}$ in particular, mono-therapy with levofloxacin has been proven to be particularly effective in animal studies where a good tissue diffusion was attained..$^{13}$ Our patient received a combination of antibiotic therapy consisting of piperacillin-tazobactam and ciprofloxacin for two weeks with good clinical response. A percutaneous drainage is successful when the abscess collection is unilocular or bilocular, with a discrete wall and no internal septation. Surgery is preferred in patients with more than two abscesses. In the event of multiple abscesses, as in our case, CTguided percutaneous drainage appears to be impractical because of the abscess size and number. However, drainage combined with antibiotic therapy may offer some advantages over splenectomy by avoiding severe complications. Thanos et al. studied eleven patients with splenic abscesses, seven of whom were treated successfully by drainage and antibiotic therapy while the remaining four underwent surgery; the favorable outcomes observed in the former group may be ascribed to the selective inclusion criteria used for drainage. ${ }^{14}$

\section{Conclusions}

Splenic abscess is a rare but extremely dangerous condition, due to the potential severity of the underlying infection, the high mortality rate and the complications of treatments. We should consider splenic abscess when a long-lasting fever and pain in the left hypochondrium are present, even when an apparently innocuous invasive procedure, such as a body piercing, is performed. Although CT remains the gold standard for the diagnosis, there is no consensus regarding standard treatment. There is increasing evidence in favor of less invasive therapeutic approaches to preserve the spleen, especially in younger patients. The combination CT-guided drainage and antibiotic therapy appears to be most beneficial in patients with solitary, well-capped abscesses. Although an exclusive antibiotic therapy consisting of beta-lactams and fluoroquinolones has been proven to be effective in our patient, more studies are needed in order to define the best therapeutic approach. 


\section{References}

1. Davido B, Dinh A, Rouveix E, et al. Abcès de la rate: du diagnostic au traitement. Rev Med Interne 2017:61418.

2. Ismail E, El Barni R, Lahkim M, et al. Splenic abscess in cancer chemotherapy. BMC Res Notes 2015;1:665.

3. Loussaief C, Toumi A, Ben Romdhane F, et al. Pyogenic splenic abscesses. A review of 8 cases. Rev Med Interne 2005;541-4.

4. Alonso Cohen MA, Galera MJ, Ruiz M, et al. Splenic abscess. World J Surg 1990;14:513-6.

5. Mushtaq H, Irfan A. Splenic Abscess of an Unknown Etiology. J Coll Physicians Surg Pak 2018;28:804-5.

6. Paris S, Weiss SM, Ayers WH, Clarke LE. Splenic abscess. Am Surg 1994;60:358-61.

7. Sarr MG, Zuidema GD. Splenic abscess-presentation, diagnosis, and treatment. Surgery 1982;92:480-5.

8. Nelken N, Ignatius J, Skinner M, Christensen N. Changing clinical spectrum of splenic abscess. A multicenter study and review of the literature. Am J Surg.1987; 154:27-34.

9. Burnier C, Ribordy-Baudat V, Lamy O. Splenic abscess: etiology, diagnosis and possible therapeutics. Praxis 2007;96:1731-5.

10. Thipphavong S, Duigenan S, Schindera ST, et al. Nonneoplastic, benign, and malignant splenic diseases: cross-sectional imaging findings and rare disease entities. AJR Am J Roentgenol 2014;203:315-22.

11. Kamaya A, Weinstein S, Desser TS. Multiple Lesions of the Spleen: Differential Diagnosis of Cystic and Solid Lesions. Semin Ultrasound CT MRI 2006;27:389-403.

12. Smyrniotis V, Kehagias D, Voros D. Splenic abscess: An old disease with new interest. Dig Surg 2000;17:354-7.

13. Zhu L, Zhang Y, Yang J, et al. Prediction of the pharmacokinetics and tissue distribution of levofloxacin in humans based on an extrapolated PBPK model. Eur J Drug Metab Pharmacokinet 2016;41:395-402.

14. Thanos L, Dailiana T, Papaioannou G, et al. Percutaneous CT-Guided Drainage of Splenic Abscess. AJR Am J Roentgenol 2002;179:629-32. 